\title{
A Fiber Optic Ultrasonic Sensor Using Polarization-maintaining Fiber for Partial Discharge Monitoring
}

\author{
Xuefeng Li, ${ }^{1}$ Hexin Cao, ${ }^{1}$ Zhiming Chang, ${ }^{2}$ \\ Xinyu Zhang, ${ }^{2}$ Weishi Zhao, ${ }^{3}$ and Runjie Shen ${ }^{1 *}$ \\ ${ }^{1}$ College of Electronic and Information Engineering, Tongji University, \\ 4800 Cao'an Highway, Shanghai 201804, P. R. China \\ ${ }^{2}$ Datang Guoxin Binhai Offshore Wind PowerCo., Ltd., \\ No. 1 Caishikou Street, Beijing 100053, P. R. China \\ ${ }^{3}$ East Branch Natural Gas Marketing Company, PetroChina Company Limited, \\ No. 1200 Century Avenue Pilot Free Trade Zone, Shanghai 200135, P. R. China
}

(Received July 4, 2018; accepted March 13, 2019)

Keywords: partial discharge, nondestructive testing, PM-PCF, PANDA fiber

Conventional acousto-ultrasonic piezoelectric sensors can be easily interfered in strong electromagnetic environments such as partial discharge (PD). Current research shows that the effective refractive index difference between two orthogonal polarization modes increases differently with external pressure. Light output intensity can be detected by interference demodulation and depolarization from the polarization-maintaining fiber (PMF) between the two orthogonal polarization modes, and then pressure variations can be obtained. In this study, a new type of acousto-ultrasonic sensor based on the principle of polarization interference is designed to detect PD-induced ultrasonic signals. Simulation and experimental results are also discussed by comparing the following two types of PMFs as the sensing unit: polarizationmaintaining photonic crystal fiber (PM-PCF) and polarization-maintaining and absorptionreducing (PANDA) fiber. Analysis results have indicated that the PM-PCF has a higher sensitivity towards external pressure, because its birefringence has a linear relationship of 2.02 $\times 10^{-6} \mathrm{RIU} / \mathrm{MPa}$, far beyond $3.01 \times 10^{-11} \mathrm{RIU} / \mathrm{MPa}$ of the PANDA fiber. Then, the function between external pressure and light output intensity can be analyzed. The proposed fiber optic sensor using the PM-PCF as the sensing unit has a higher signal-to-noise ratio (SNR). Therefore, this type of sensor can more easily find the initial failure of equipment.

\section{Introduction}

Gas-insulated switchgear (GIS) has various advantages such as an integrated structure, a small electromagnetic interference, and low-cost maintenance. It has been used widely in current extra-high-voltage (EHV) distribution and transmission systems. ${ }^{(1,2)}$ However, GIS faults without prior detection can result in the destruction of large-area grid systems and in

*Corresponding author: e-mail: shenrunjie@tongji.edu.cn https://doi.org/10.18494/SAM.2019.2260 
extraordinary economic losses. The reliability of insulation in the GIS is regarded as one of the most important characteristics in monitoring the health status of EHV systems. ${ }^{(3-5)}$ Partial discharge (PD) is usually an early signal of insulation failure. It often occurs with secondary physical phenomena such as light and sound generation, temperature fluctuation, electromagnetic wave emission, and chemical reactions. ${ }^{(6-10)}$ By measuring these relative performance characteristics of PD, potential internal threats can be detected so that GIS faults can be recognized and eliminated in advance.

Being a nondestructive testing technology, acousto-ultrasonic detection can be fully applied in monitoring the structural stress destruction and discharge damage of insulating materials. ${ }^{(11,12)}$ However, conventional acousto-ultrasonic sensors are mainly piezoelectric sensors. These sensors are manufactured using the principle of piezoelectric induction, so they are easily interrupted and hardly maintain a high reliability in strong electromagnetic environments such as PD in EHV systems. ${ }^{(11,13-15)}$ As a result, in order to design a new type of acousto-ultrasonic sensor to satisfy the requirements of high stability and reliability in the long-period detection of a harsh environment, another measurement principle or material should be considered and developed.

Fiber optic sensors have been used widely in modern industry, which provides an ideal solution to this problem. There are many advantages of fiber optic sensors such as small volume, tolerance to high pressure and temperature, and immunity to electromagnetic interference. ${ }^{(16,17)}$ Fiber optic sensors are based on the following different principles: intensity modulation, phase modulation, polarization modulation, frequency modulation, wavelength modulation, and so on. ${ }^{(18-20)}$ However, there are two types of sensors that receive much more attention, namely, phase and wavelength modulation sensors. Both of them have the advantages of high reliability and stability. However, phase modulation sensors do not have fast responses towards signals, and the structure of wavelength modulation sensors is usually complex, which may induce a strong noise. Current research indicates that the effective refractive index difference between the two orthogonal polarization modes increases differently with external pressure. Light output intensity can be detected by interference demodulation and depolarization from the polarization-maintaining fiber (PMF) between the two orthogonal polarization modes, and then pressure variations can be obtained. Before this research, one type of acoustic-emission sensor based on the principle of polarization modulation has been designed by our research team to detect PD-induced pressure signals. ${ }^{(21)}$ However, the study on the characteristics of sensor sensitivity is pending to be further improved.

In this study, two acousto-ultrasonic sensors based on the same principle of polarization interference are fabricated to monitor PD-induced ultrasonic signals. Firstly, a simulation based on the finite element method (FEM) is performed to compare the following two types of PMFs as the sensing unit: polarization-maintaining photonic crystal fiber (PM-PCF) and polarizationmaintaining and absorption-reducing (PANDA) fiber. Analysis results indicate that the PMPCF has a higher sensitivity towards external pressure, because its birefringence has a linear relationship of $2.02 \times 10^{-6} \mathrm{RIU} / \mathrm{MPa}$, far beyond $3.01 \times 10^{-11} \mathrm{RIU} / \mathrm{MPa}$ of the PANDA fiber. Then, the new sensor is designed and the experimental platform is established to test 
its performance. Results prove that the proposed fiber optic sensor using the PM-PCF as the sensing unit has a higher signal-to-noise ratio (SNR).

\section{Sensing Principle}

Figure 1 shows the structure of the fiber optic ultrasonic sensor using the PMF. First, the light from the laser source is polarized through the polarizer. The initial intensities in the $x$ and $y$-directions should be equal so that the final intensity can be calculated. Thus, the light is injected $45^{\circ}$ to the slow axis ( $x$-axis) of the sensing unit, which is composed of the PMF. Then, the sensing unit decomposes the incident light into two perpendicular linearly polarized lights, $E_{x}$ and $E_{y}$, according to different propagation constants, $\beta_{x}$ and $\beta_{y}$. The fiber splitter distinguishes the two beams of polarized light, and sends them to the fiber coupler, which rotates one of the polarization directions by $90^{\circ}$ and combines two polarized lights. Finally, the unit detector receives the coupled signal and sends it to the signal acquisition terminal for processing and analyis.

Equation (1) describes the output light intensity, where $k_{0}=2 \pi / \lambda$ is the wave number in vacuum and $L$ is the length of the fiber. In this research, the wavelength $\lambda$ is $1550 \mathrm{~nm}$, so $k_{0}$ is $4.05 \times 10^{6} \mathrm{rad} / \mathrm{m}$.

$$
\begin{aligned}
I & =\left|E_{x}+E_{y}\right|^{2} \\
& =\left|E_{0} \cdot e^{-j \Phi_{1}}+E_{0} \cdot e^{-j \Phi_{2}}\right|^{2} \\
& =E_{0}^{2} \cdot\left|\cos \Phi_{1}+\cos \Phi_{2}-j\left(\sin \Phi_{1}+\sin \Phi_{2}\right)\right|^{2} \\
& =2 E_{0}^{2} \cdot\left(1+\cos \left(k_{0} L B\right)\right) \\
\Phi_{1} & =\frac{2 \pi L \cdot n_{x}}{\lambda}=k_{0} n_{x} L, \Phi_{2}=\frac{2 \pi L \cdot n_{y}}{\lambda}=k_{0} n_{y} L
\end{aligned}
$$

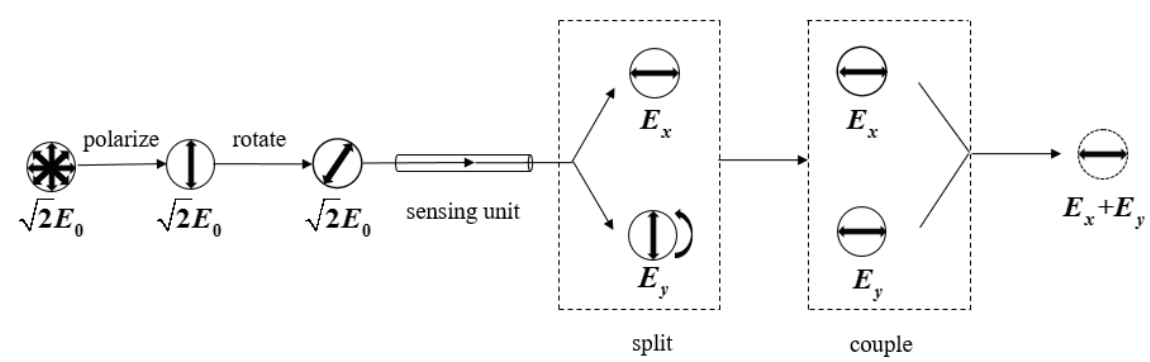

Fig. 1. Structure of the fiber optic ultrasonic sensor. 
$\Phi_{1}$ and $\Phi_{2}$ in Eq. (2) are the differences between the phase of the fiber output and the initial phase. $n_{x}$ and $n_{y}$ are the effective refractive indices of $x$ - and $y$-polarized lights, respectively. The relationship between $n_{x}, n_{y}$, and the propagation constant is $\beta=k_{0} n_{\text {eff. }} \quad B=\left|n_{x}-n_{y}\right|=$ $k_{0}\left|\beta_{x}-\beta_{y}\right|$, defined as the birefringence, is the key factor to the output interference light intensity. When the PMF is under external pressure, the optical properties change because of the photoelastic effect, result in the change in $B$, and consequently affect the output light intensity. ${ }^{(22)}$

\section{Simulation}

In this paper, FEM is used as the method of analysis for the sensor in simulation. COMSOL Multiphysic $^{\circledR}$ is used here to analyze the birefringent properties of the PMF under external pressure. $^{(23-25)}$ The SM15-PS-U25D PANDA fiber fabricated by Japan Fujikura Ltd. and the PM-1550-01 PM-PCF produced by NTK Photonics Inc. are adopted as the sensing unit and analyzed separately. The sectional images of the two PMFs are shown in Figs. 2(a) and 2(b), respectively.

In this simulation, the stress distribution induced by the ultrasonic signal and its effect on the mode effective refractive index from the section of fibers are investigated; therefore, a 2D space dimension is chosen as the cross-sectional model of the sensing unit. Figures 3(a)-3(d) are the polarization modes $x$ and $y$ of the two fibers when the wavelength is $1550 \mathrm{~nm}$. The mode field shrinks in the core, which proves that the two polarization modes are orthogonal to each other and the fundamental mode energy transmission is in the normal state.

The PM-PCF is made of only silicon, so there is almost no internal stress when the temperature is from the annealing level $\left(1920^{\circ} \mathrm{C}\right)$ to a normal value. However, the PANDA fiber has a larger coefficient of thermal expansion in the stress zone. When the temperature decreases to the normal value, the difference in the coefficient of thermal expansion generates a strong stress distribution inside. Thus, when pressure is set on the outside of the fiber, part of it will be used to offset the internal stress of the fiber. Therefore, the sensor using the PMPCF has a higher sensitivity towards pressure signals induced by ultrasonic waves. Figure 4(a) shows the relationship between the effective refractive index $\left(n_{\text {eff }}\right)$ and the centripetal pressure of the two polarization modes. Therefore, once PD occurs, the birefringence changes lead to

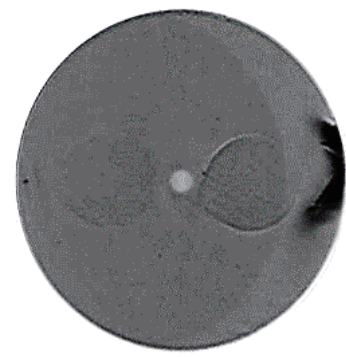

(a)

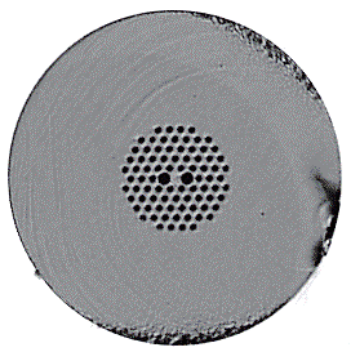

(b)

Fig. 2. Sectional images of (a) SM15-PS-U25D PANDA fiber and (b) PM-1550-01 PM-PCF. 


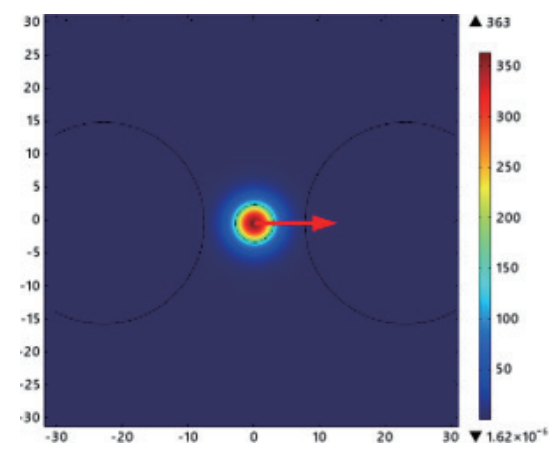

(a)

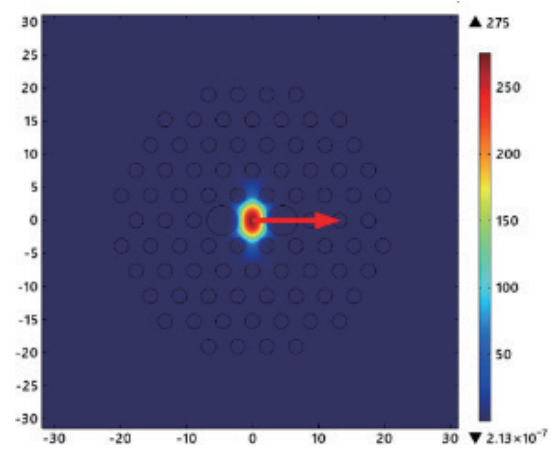

(c)

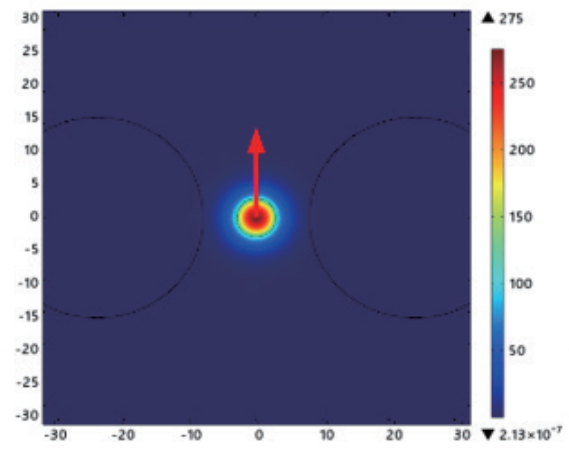

(b)

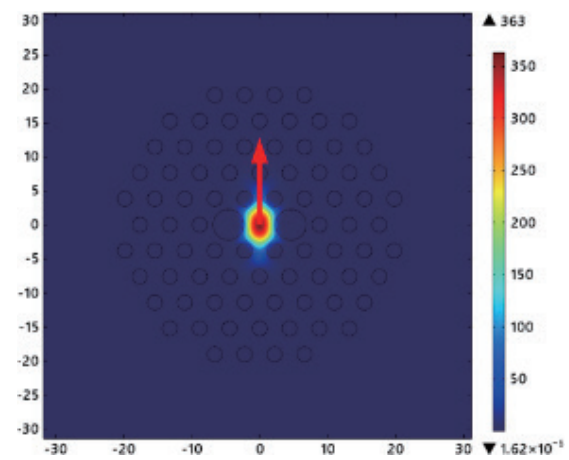

(d)

Fig. 3. (Color online) (a) Polarization mode $x$ of SM15-PS-U25D PANDA fiber, (b) polarization mode $y$ of SM15PS-U25D PANDA fiber, (c) polarization mode $x$ of PM-1550-01 PM-PCF, and (d) polarization mode $y$ of PM-155001 PM-PCF.

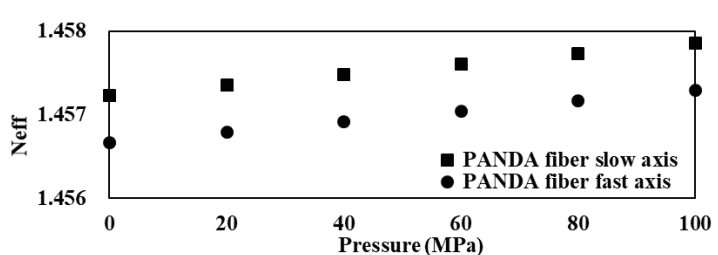

(a)

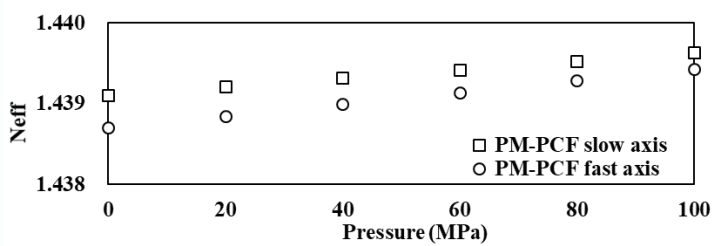

(b)

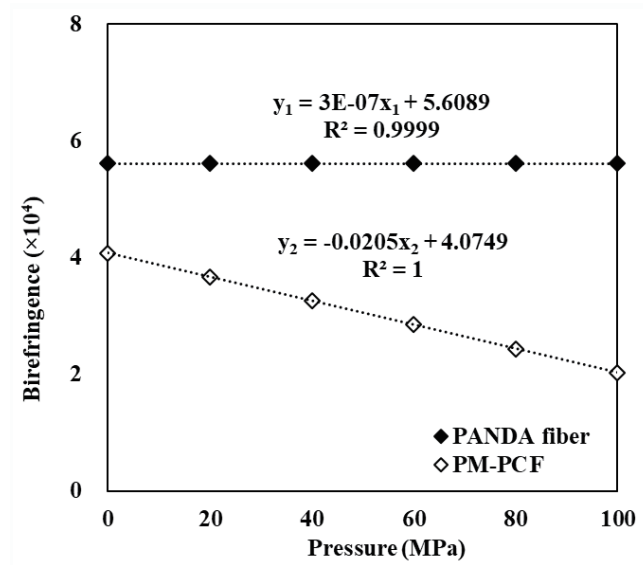

(c)

Fig. 4. (a) Relationship between effective refractive index ( $\left.n_{\text {eff }}\right)$ and centripetal pressure of PANDA fiber, (b) relationship between effective refractive index $\left(n_{e f f}\right)$ and centripetal pressure of PM-PCF, and (c) birefringent properties of PANDA fiber and PM-PCF. 
the variation in light output intensity. As a result, pressure variations induced by ultrasonic waves can be calculated by interference demodulation between the $x$ - and $y$-polarization modes. Figure 4(b) shows the birefringent properties of the PANDA fiber and PM-PCF. It can be inferred that the PM-PCF has a much higher sensitivity towards centripetal pressure, because its birefringence has a linear relationship of $2.02 \times 10^{-6} \mathrm{RIU} / \mathrm{MPa}$, far beyond $3.01 \times$ $10^{-11}$ RIU/MPa of the PANDA fiber. Figures 5(a) and 5(b) show the relationship between the light output intensity and the external pressure of the two PMFs, indicating that the light output intensity of the PM-PCF experiences much more cycles in the unit pressure than that of the PANDA fiber. Simulation and theoretical results show that the PM-PCF proves to be a better type of PMF as the sensing unit in PD detection. The following part will indicate that it still has advantages in an actual measurement through experiments.

\section{Experiment}

In the experiment, two types of PMF are used as the sensing unit, and the traditional singlemode silica fiber is used as the signal transmission unit. Since the silica fiber and PM-PCF have large differences in geometry, the fiber fusion method is adopted to reduce the connection loss. An important problem in the process of sensor-sensitive unit fabrication is the splice loss. The main reason for this is that the fusion point of the PM-PCF is lower than that of the traditional silica fiber, so the intensity and period of discharging in the splicing process for the silica fiber are very large, which causes the collapse of air holes and the destruction of the photonic crystal structure. To minimize the splicing loss, the discharge strategy of the fiber splicer should be adjusted. Aiming to solve this problem, firstly, the focus mode is set as an outer diameter mode. The diameter of a bare fiber for both the PM-PCF and the silica fiber is $125 \mu \mathrm{m}$; the two fibers are exactly the same when stripped with the coating layer and outer cladding, and their core areas are both at the center. Therefore, the outer diameter focus mode can minimize the shift loss. Secondly, we decrease the intensity and period of discharge, and set the center position of the electrode on the side near the traditional silica fiber. This discharge strategy will distribute

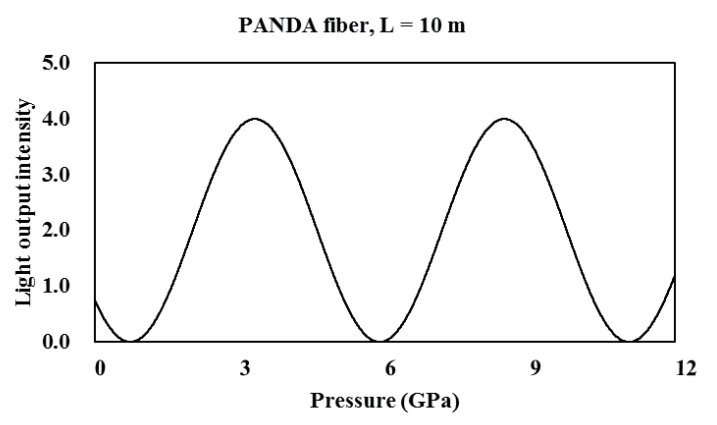

(a)

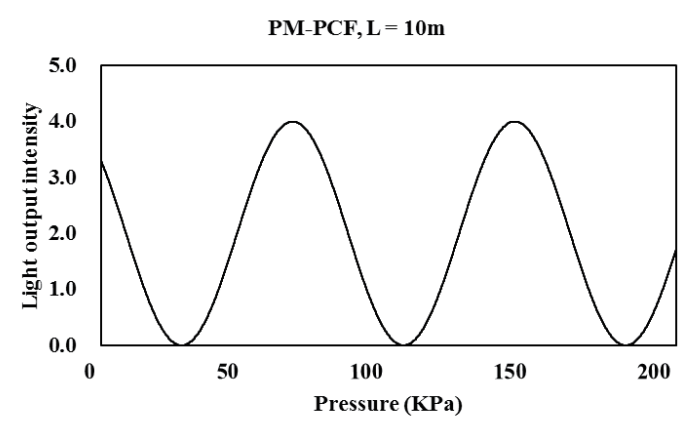

(b)

Fig. 5. (a) Relationship between output optical power and pressure of PANDA fiber. (b) Relationship between output optical power and pressure of PM-PCF. 
less energy at the end of the PM-PCF to avoid the collapse of its air holes, while distributing more energy at the end of the silica fiber to obtain a better fusion state with a lower discharge intensity. Figure 6(a) shows the image of the fusion of two fibers. The right part of the PM-PCF is blurred because it is made of only one material (silicon), and there are only air holes inside it to guide the core of the fiber. The left fiber is clear because the core of the silica fiber is mixed with other chemical elements. By measuring the loss of the sensing unit (two fusion points), the loss value is about $-10 \mathrm{~dB}$.

Figure 6(b) shows the image of the platform that contains the fiber optic measurement system and disc-type insulator. The measurement system is composed of the following eight parts: (1) YW-B430i benchtop laser source, (2) ILP-1550-SM-PM-900-1-FC fiber polarizer, (3) MJP-1550-900-0.5-FU fiber optic rotator, (4) PANDA fiber/PM-PCF sensing unit, (5) PBS1550-P-000-3-2-1-1 fiber polarization beam splitter, (6) PMFC-1*2-1550-50/50-900-FU fiber coupler, (7) PDA1008 unit detection module, and (8) PCI-1714UL signal acquisition terminal. The insulator, which is made of epoxy resin, is used as the carrier for PD tests. The PMF is embedded in the U-shaped groove of the insulator by the insulation tape (black areas) as the sensing unit. First, the laser produces the unpolarized light. The light is polarized by the fiber polarizer and then rotated $45^{\circ}$ to the slow axis of the PMF by the rotator. Therefore, two mutually perpendicular linearly polarized lights are induced in the sensing unit. The fiber splitter decouples the two beams of the polarized light and sends them to the fiber coupler. The coupled signal from the coupler is sent to the unit detector module, which is connected to the data acquisition card, and finally analyzed by the terminal. The needle-plate discharge model is adopted to generate the PD signals.

In Table 1, two types of fiber optic ultrasonic sensor are compared. The discharge voltage can be recorded when the tip of the needle produces intermittent discharge to the insulator. To identify the performance of the sensors, the arcing distance is set from 1 to $5 \mathrm{~mm}$, while the discharge voltage varies from 2.9 to $13.2 \mathrm{kV}$. The experimental results indicate that the proposed sensor using the PM-PCF as the sensing unit has a higher SNR than the PANDA fiber,

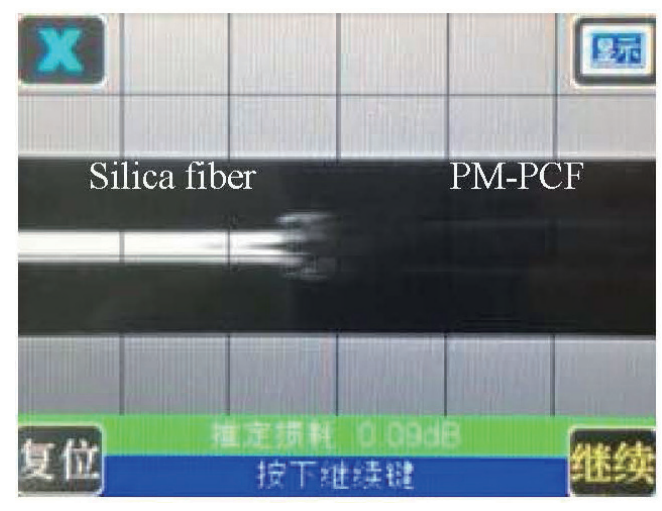

(a)

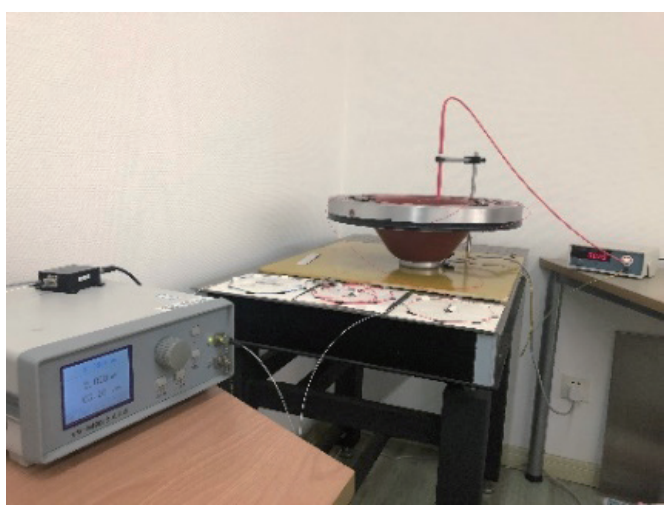

(b)

Fig. 6. (Color online) Images of (a) the fusion of two fibers and (b) the platform. 
Table 1

Comparison of two types of fiber optic ultrasonic sensor.

\begin{tabular}{lccccc}
\hline Discharge spacing & $5 \mathrm{~mm}$ & $4 \mathrm{~mm}$ & $3 \mathrm{~mm}$ & $2 \mathrm{~mm}$ & $1 \mathrm{~mm}$ \\
(Discharge voltage) & $(13.2 \mathrm{kV})$ & $(11.4 \mathrm{kV})$ & $(9.2 \mathrm{kV})$ & $(8.1 \mathrm{kV})$ & $(2.9 \mathrm{kV})$ \\
\hline
\end{tabular}
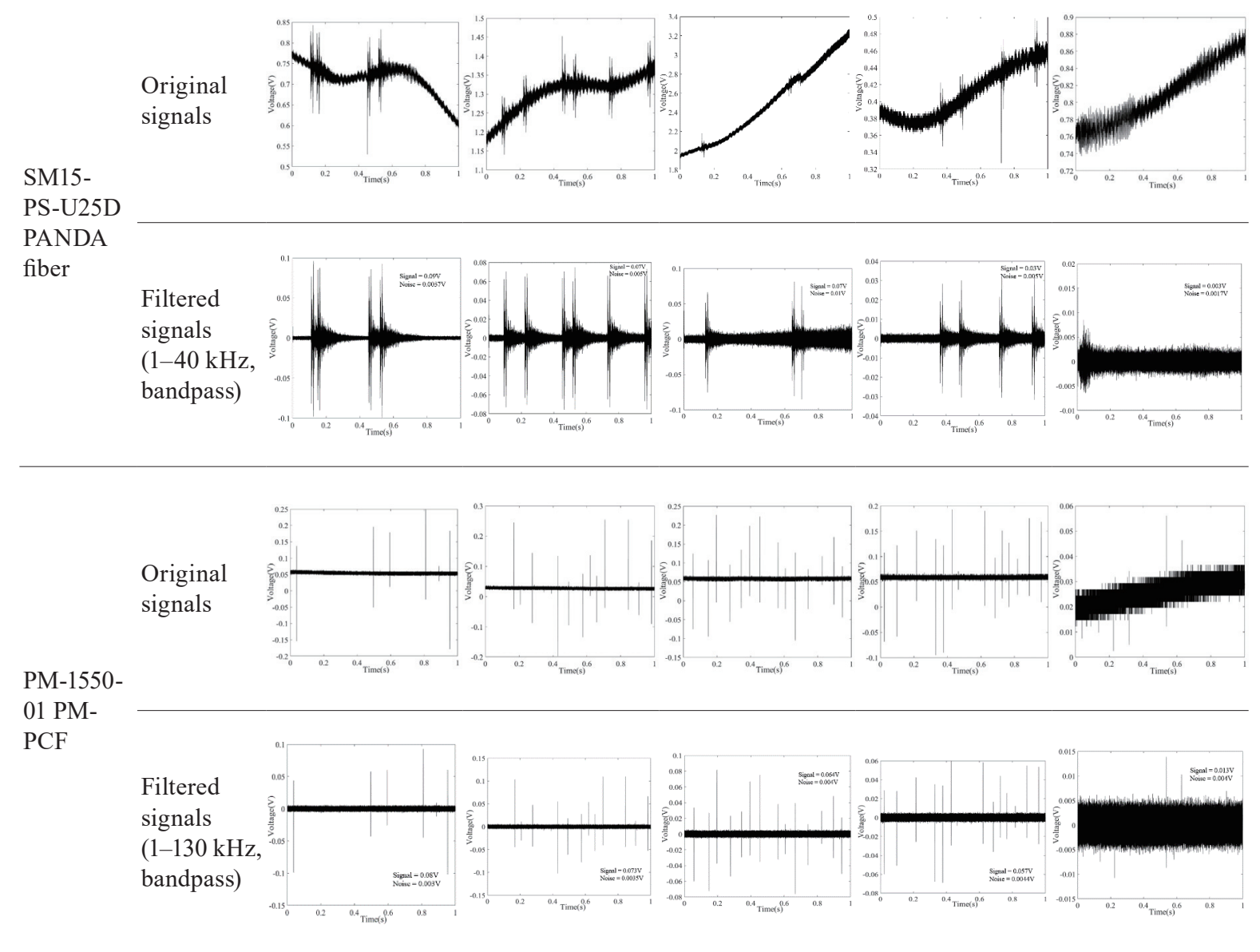

which is compatible with the simulation results, indicating that the PM-PCF shows a higher performance in PD detection. One thing to note here is that the bandpass filter is used here to reduce noise interference. Many experiments indicate that, when using the PANDA fiber as the sensing unit, setting the bandpass range to $1-40 \mathrm{kHz}$ can better filter out environmental noise. The same is true for the PM-PCF. Therefore, the parameters of the two filters are different: one of the passbands is $1-40 \mathrm{kHz}$, while the other is $1-130 \mathrm{kHz}$. The acquisition of signals and noise is shown in Fig. 7(a). Here, the noise is mainly caused by the dark current in the unit detection module. Figure 7(b) shows the SNRs of the two types of sensor. The SNRs improve with increasing discharge spacing (increasing discharge voltage). The performance of the sensor based on the PM-PCF is higher than that of the sensor based on the PANDA fiber in all measurement ranges; particularly when measuring small signals, the PM-PCF sensor has superior characteristics. It can detect early equipment failures when PD-induced ultrasonic signals are not obvious. 


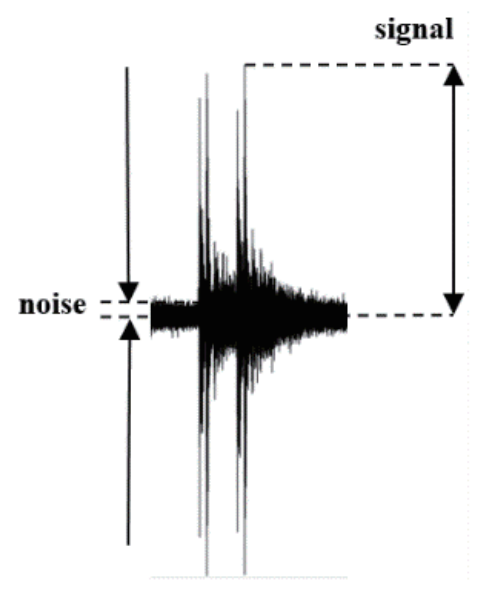

(a)

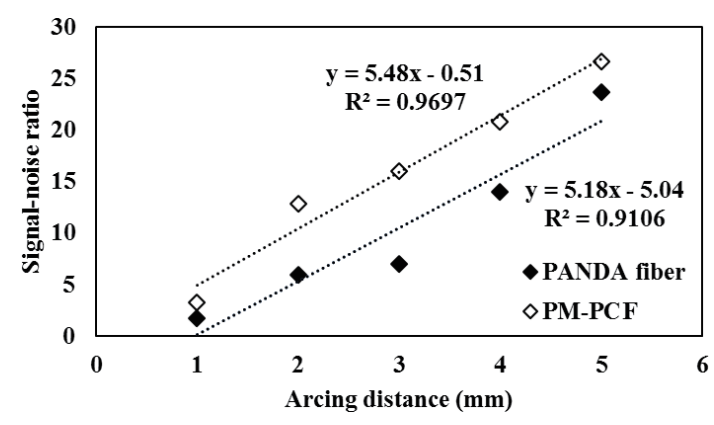

(b)

Fig. 7. (a) Acquisition of signals and noise. (b) Comparison of the SNRs of two sensors.

\section{Conclusions}

In this paper, two types of fiber optic ultrasonic sensor using various PMFs are compared and analyzed for the ultrasonic detection of GIS faults. The simulation based on FEM enables the evaluation of the performance of the sensor. A comparison of the two PMFs is also discussed and simulation results show that the PM-PCF has a higher sensitivity towards ultrasonic signals, because its birefringence has a linear relationship of $2.02 \times 10^{-6} \mathrm{RIU} / \mathrm{MPa}$, far beyond $3.01 \times 10^{-11} \mathrm{RIU} / \mathrm{MPa}$ of the PANDA fiber. Then, an experimental platform is set up. The sensing unit is embedded in the disc-type insulator, and the needle plate discharge model is used to generate PD-induced signals. The sensor using the PM-PCF shows a much higher signal response after being processed with a bandpass filter, which is in accordance with simulation results. The proposed sensor, with the PM-PCF as the sensing unit, can satisfy the need for detecting ultrasonic signals within its frequency range. It has many advantages such as a small volume, high sensitivity and stability, and resistance to electromagnetic interference. This type of fiber optic ultrasonic sensor can be widely used for monitoring in different fields such as PD detection in a high-voltage environment, the structural destruction of an engineering building, and seismic wave monitoring in earthquakes. Further research will focus on the improvement of the measurement system in order to reduce noise caused by dark current.

\section{Acknowledgments}

This work is partially supported by the Fundamental Research Funds for the Central Universities (22120180121, 22120170260, and 22120180299) and the National Natural Science Foundation of China (Grant No. 61873189). 


\section{References}

1 Y. Liu, H. Hong, X. Lu, S. Li, Y. Hou, J. Zhang, G. Liu, and J. Zhang: 4th Int. Conf. Electric Power EquipmentSwitching Technology (ICEPE-ST, 2017) 272-274.

2 T. Linn: 2016 IEEE Int. Conf. High Voltage Engineering and Application (ICHVE) (IEEE, 2016) 1-4.

3 H. Chang, F. Gu, and C. Kuo: 2012 IEEE Int. Power Modulator and High Voltage Conf. (IPMHVC) (IEEE, 2012) 165-168.

4 P. Kaur and T. Choudhury: 7th India Int. Conf. Power Electronics (IICPE) (IEEE, 2016) 1-6.

5 M. Hu, H. Wang, Y. Chang, and X. Pan: 4th Int. Conf. on Appl. Robotics for the Power Industry (CARPI) (IEEE, 2016) $1-6$.

6 C. Chou and C. Chen: 7th Int. Symp. Next Generation Electronics (ISNE) (IEEE, 2018) 1-4.

7 G. C. Stone, M. K. W. Stranges, and D. G. Dunn: IEEE Ind. Appl. Mag. 22 (2016) 14.

8 M. Florkowski, B. Florkowska, and P. Zydron: 2016 IEEE Int. Conf. High Voltage Engineering and Application (ICHVE, 2016) 1-4.

9 C. Thirumurugan, G. B. Kumbhar, and R. Oruganti: 11th Int. Conf. Properties and Applications of Dielectric Materials (ICPADM, 2015) 428-431.

10 S. M. Gargari, P. A. A. F. Wouters, P. C. J. M. van der Wielen, and E. F. Steennis: IEEE Trans. Dielectrics and Electrical Insulation. 18 (2011) 868.

11 G. Wild and S. Hinckley: IEEE Sens. J. 8 (2008) 1184.

12 M. Anaya, D. A. Tibaduiza, E. Forero, R. Castro, and F. Pozo: 20th Symp. Signal Processing, Images and Computer Vision (STSIVA, 2015) 1-5.

13 A. Bulletti, P. Giannelli, M. Calzolai, and L. Capineri: IEEE Trans. Ultrason. Ferroelectr. Freq. Control. 63 (2016) 864.

14 K. R. Rajesh, R. Murali, and R. Mohanachandran: 2011 IEEE Global Humanitarian Technol. Conf. (IEEE, 2011) 316-321.

15 L. Jerabek, A. L. Bartos, and J. Strycek: 2011 IEEE Int. Conf. Acoustics, Speech and Signal Processing (ICASSP) (IEEE, 2011) 1793.

16 O. Sidek and M. H. Bin Afzal: 2011 IEEE Symp. Business, Engineering and Industrial Applications (ISBEIA) (IEEE, 2011) 458-463.

17 C. Wu, X. Zhang, and Z. Huang: 15th Int. Conf. Optical Communications and Networks (ICOCN) (IEEE, 2016) 1-3.

18 J. Hervás, D. Barrera, J. Madrigal, and S. Sales: 25th Optical Fiber Sensors Conf. (OFS) (IEEE, 2017) 1-4.

19 X. Jin, J. Ou, R. Hao, F. Zhou, X. Zhang, S. Zheng, and H. Chi: IEEE Photonics Technol. Lett. 24 (2012) 1981.

20 A. V. Volkov, M. Y. Plotnikov, M. V. Mekhrengin, G. P. Miroshnichenko, and A. S. Aleynik: IEEE Sens. J. 17 (2017) 4143.

21 X. Li, Y. Zhang, H. Cao, X. Zhang, and R. Shen: Sens. Mater. 30 (2018) 1145.

22 S. Revathi, S. R. Inabathini, and J. Pal: OPTIK 126 (2015) 3395.

23 S. Azzam and S. S. A. Obayya: 31st Int. Rev. Prog. Appl. Comput. Electromagnet. (ACES, 2015) 1-2.

24 T. Mifune: 2016 IEEE/ACES Int. Conf. Wireless Information Technol. and Systems and Appl. Computational Electromagnetics (ICWITS and ACES) (IEEE, 2016) 1-4.

25 M. E. Royak and E. R. Lyzov: 13th Int. Sci. Tech. Conf. Actual Problems of Electronics Instrument Engineering (APEIE) (IEEE, 2016) 327-329.

\section{About the Authors}

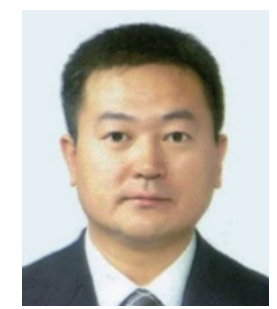

Xuefeng Li graduated from Shenyang Institute of Engineering, China, in 1999. He received his M.E. and D.E. degrees from Fukuoka Institute of Technology, Fukuoka, Japan, in 2004 and 2007, respectively. From 2007 to 2013, he was a postdoctorate and assistant researcher at Waseda University, Kitakyushu, Japan. Since 2010, he has been an associate professor at Tongji University. His research interests are in sensors and NDT. 


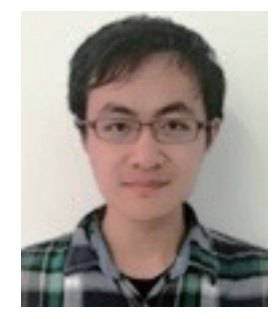

Hexin Cao received his B.S. degree from HeFei University of Technology, China, in 2016. Since 2016, he has been studying at Tongii University for his M.S. degree. His research interests are in sensors and NDT.

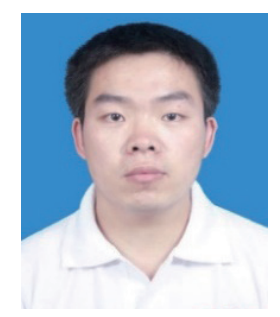

Zhiming Chang graduated from Changchun Institute of Technology, China, in 2009. He entered China Datang Corporation in 2009. Since 2017, he has been the deputy director of the Safety Production Department at Datang Guoxin Binhai Offshore Wind Power Co., Ltd. His research interests are in new energy generation technology.

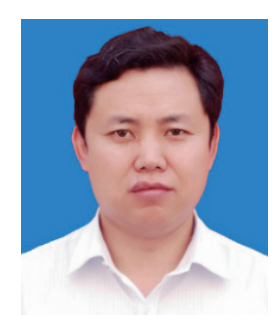

Xinyu Zhang received his B.S. degree from Northeast Electric Power University, China, in 1997. Since 2016, he has been the chief engineer at the East China branch of China Datang Corporation Renewable Power Co., Ltd.

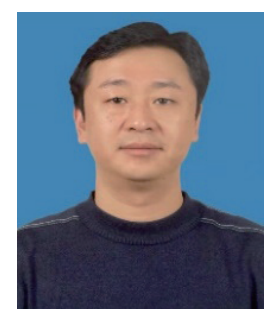

Weishi Zhao received his B.S. degree from Changchun University of Technology, China, in 2008. In 1995, he entered Changchun Oil Transportation Communication Company. Since 2018, he has been the chief engineer at the East China branch of China National Petroleum Corporation.

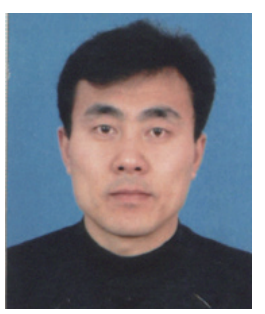

Runjie Shen received his B.S. degree from Xi'an Polytechnic University, China, in 1995. He obtained his M.S. degree from Xi'an Jiaotong University, China, in 1998 and his Ph.D. degree from Zhejiang University, China, in 2004. From 2004 to 2006, he was a postdoctorate at Zhejiang University. In 2006, he became an associate researcher at Zhejiang University. Since 2011, he has been an associate researcher at Tongji University. His research interests are in sensors and NDT. 